\title{
The Immunobiology of Inductive Anti-CD40L Therapy in Transplantation: Allograft Acceptance is Not Dependent Upon the Deletion of Graft-Reactive T Cells
}

\author{
Meera J. Nathanb, Dengping Yinc, \\ Ernst J. Eichwald ${ }^{d}$ and D. Keith Bishop ${ }^{a, b}$,* \\ a Division of Transplantation, Department of Surgery and \\ ${ }^{b}$ Department of Microbiology and Immunology, University \\ of Michigan School of Medicine, Ann Arbor, MI 48109; \\ USA \\ ${ }^{c}$ Department of Surgery, Section of Transplantation, Rush \\ Presbyterian St. Luke's Medical Center, Chicago, IL; USA \\ ${ }^{d}$ Department of Pathology, University of Utah School of \\ Medicine, Salt Lake City, UT 84132, USA \\ ${ }^{*}$ Corresponding author: D. Keith Bishop, \\ kbishop@umich.edu
}

CD40-CD40L costimulatory interactions are crucial for allograft rejection, in that treatment with anti-CD40L mAb markedly prolongs allograft survival in several systems. Recent reports indicate that costimulatory blockade results in deletion of graft-reactive cells, which leads to allograft tolerance. To assess immunologic parameters that were influenced by inductive CD40-CD40L blockade, cardiac allograft recipients were treated with multiple doses of the anti-CD40L mAb MR1, which was remarkably effective at prolonging allograft survival. Acute allograft rejection responses such as IL-2 producing helper cell priming, Th1 priming, and alloantibody production were abrogated by anti-CD40L treatment. Interestingly, the spleens of mice bearing long-term cardiac allografts following inductive anti-CD40L treatment retained precursor donor alloantigen-reactive CTL, IL-2 producing helper cells, and Th1 in numbers comparable to those observed in naive mice. These mice retained the ability to reject donor-strain skin allografts, but were incapable of rejecting the original cardiac allograft, or a second donor-strain cardiac allograft. Further, differentiated effector cells were incapable of mediating rejection following adoptive transfer into mice bearing longterm allografts, suggesting that regulatory cell function, rather than effector cell deletion was responsible for long-term graft acceptance. Collectively, these data demonstrate that inductive CD40-CD40L blockade does not result in the deletion of graft-reactive $\mathrm{T}$ cells, but induces the maintenance of these cells in a quiescent precursor state. They further point to a tissue specificity of this hyporesponsiveness, suggesting that not all donor alloantigen-reactive cells are subject to this regulation.

Key words: Costimulation, graft acceptance, regulatory cells
Received 24 May 2001, revised and accepted for publication 17 December 2001

\section{Introduction}

It has long been appreciated that two signals are required for T-cell activation (1). The first signal results from the engagement of the T-cell receptor (TCR) with the major histocompatibility complex (MHC)-peptide complex on the antigen-presenting cell (APC), and the second signal involves engagement of costimulatory receptors on the $T$ cell with their respective ligands on the APC. Absence of the second signal results in either T-cell anergy (1) or deletion of these cells $(2,3)$. The two best-characterized costimulatory pathways are those involving CD28-CD80/86 and CD40-CD40 ligand (CD40L) interactions. Both CD28-CD80/86 and CD40CD40L interactions are critical in T-cell priming, maturation, and activation of the APC (4). Hence, the importance of the CD28-CD80/86 and CD40-CD40L pathways in allograft rejection has been explored by a number of investigators [reviewed in $(5,6)]$

It is not fully understood how blockade of costimulatory molecules results in allograft acceptance. A number of mechanisms, including deletion and/or anergy of donor-reactive cells, as well as the emergence of regulatory cells, have been proposed $(2,3)$. CD28 is constitutively expressed on naive $T$ cells, and engagement of the CD40-CD40L pathway is believed to be required for the up-regulation of CD80/86, CD28's cognate receptor(s) on the APC. Subsequent engagement of the CD28-CD80/86 pathway results in the activation of the APC as well as the T cell (4). Thus, blockade of the 'upstream' CD40-CD40L pathway is thought to prevent T cells from receiving the requisite costimulatory signal through CD28 (4), thus rendering the T cells anergic (1). However, others have suggested that anti-CD40L treatment directly transmits an immunomodulatory signal to the T cell, leading to apoptosis of the cell, which cannot be reversed by CD28 stimulation (7). In addition, deletion of donor-reactive cells resulting from costimulatory blockade cells has been proposed as a necessary step in achieving transplantation tolerance $(2,3)$. Indeed, recent reports indicate that apoptosis of donor-reactive $T$ cells following inductive CD40L blockade and treatment with CTLA4-lg is required for the establishment of tolerance $(8,9)$. Finally, anti-CD40L therapy has recently been shown to induce regulatory cells that are capable 


\section{Nathan et al.}

of facilitating infectious tolerance (10). Hence, the current study was aimed at further elucidating the mechanisms by which inductive anti-CD40L therapy promotes long-term allograft acceptance. This report demonstrates that inductive CD40-CD40L blockade does not result in the deletion of donor alloantigen-reactive cells. Rather, graft acceptance is associated with the maintenance of the cells in a quiescent, precursor state. The potential induction and function of regulatory cells following anti-CD4OL therapy is discussed.

\section{Materials and Methods}

\author{
Mice \\ Female C57BL/6 and BALB/c mice were obtained from Jackson Labora- \\ tories (Bar Harbor, ME, USA), and used between 6 and 12 weeks of age.
}

\section{Media}

The culture medium used in these studies was DMEM supplemented with $0.27 \mathrm{~mm}$ L-asparagine, $1.4 \mathrm{~mm} \mathrm{~L}$-arginine $\mathrm{HCl}, 14 \mathrm{~mm}$ folic acid, $5 \times 10^{-5} \mathrm{M}$ 2-ME (all obtained from Sigma Chemical, St. Louis, MO, USA), $1.6 \mathrm{~mm} \mathrm{L-}$ glutamine, $10 \mathrm{~mm}$ HEPES buffer, $1.0 \mathrm{~mm}$ sodium pyruvate, 100 units $/ \mathrm{mL}$ penicillin/streptomycin, 2\% fetal calf serum (FCS) (all obtained from Life Technologies, Grand Island, NY, USA).

\section{Heterotopic cardiac transplantation}

C57BL/6 $\left(\mathrm{H}-2^{\mathrm{b}}\right)$ mice were transplanted with intact BALB/c $\left(\mathrm{H}-2^{\mathrm{d}}\right)$ cardiac allografts, as described (11). In this model, the donor heart is anastomosed to the great vessels of the abdomen, is perfused with the recipient mouse's blood and resumes contraction. Transplant function is monitored by daily abdominal palpation.

A group of mice that had accepted their abdominal cardiac allografts following inductive anti-CD4OL treatment were transplanted with a second BALB/C cardiac allograft in the cervical position, as described (12), with slight modifications. Briefly, end-to-side anastomoses were performed between the donor heart's aorta and pulmonary artery and the recipient's carotid artery and jugular vein, respectively. In this model, allograft function was monitored visually for contraction.

\section{Skin grafting}

Skin (approximately $1 \mathrm{~cm}^{2}$ in diameter) was prepared from the abdominal region of either allogeneic BALB/c or syngeneic C57BL/6 donors. Skin was excised from the recipients' upper torso and the donor skin was then placed over the incision and held in place with wound clips. Clips were removed 3 days post-skin transplantation, and skin grafts were monitored daily. Skin grafts were considered rejected when the majority of the graft tissue was shed.

\section{Inductive anti-CD4OL therapy}

Anti-CD40L mAb (hybridoma MR1, kindly provided by Dr Randy Noelle, Dartmouth College) was purified by Lygocyte Pharmaceuticals (Bozeman, MT, USA). Biologic activity of anti-CD4OL preparations was verified in mixed lymphocyte cultures, where maximal inhibition of proliferation was achieved at $100 \mu \mathrm{g} / \mathrm{mL}$. Allograft recipients were injected i.p. with $1 \mathrm{mg}$ of anti-CD4OL on days $0,1,2,4,6,8,10$ (7 mg total), or on days $0,1,2$ (3mg total) after transplantation. Both MR1 regimens resulted in uniform prolonged graft survival ( $>60$ days) and identical immune responsiveness. Control allograft recipients were either given equivalent amounts of hamster IgG (h-lgG, Accurate Chemicals, Westbury, NY, USA) or were left untreated.
Adoptive transfer of differentiated effector cells into mice bearing long-term cardiac allografts

Spleens were harvested 10 days post transplantation from unmodified allograft recipients that had acutely rejected their grafts. Spleens were pooled and processed into single-cell suspensions, and $5 \times 10^{7}$ splenocytes were injected i.v. into mice bearing long-term (day 60) cardiac allografts. These splenocytes were also injected into SCID allograft recipients as a positive control for their ability to mediate graft rejection.

\section{Histology}

Fragments of cardiac allografts were fixed in 10\% neutral formalin, embedded in paraffin, sectioned at $5 \mu \mathrm{m}$, and stained with hematoxylin and eosin (H\&E) for examination by light microscopy. Where indicated, sections were processed for trichrome or elastin staining to detect collagen deposition and neointimal development, respectively. Parameters assessed included the intensity and composition of cellular infiltration into the grafts, myocyte death, as determined by nuclear degradation and by loss of cross striation, and inflammation of the coronary vessels.

\section{Assessment of primed and precursor Th1 function}

To monitor primed Th1 function, splenocytes $\left(1 \times 10^{6}\right.$ cells $\left./ \mathrm{mL}\right)$ isolated from allograft recipients were stimulated with irradiated (5000R) donor strain splenocytes $\left(1 \times 10^{6}\right.$ cells $\left./ \mathrm{mL}\right)$ for $72 \mathrm{~h}$, and the concentration of interferon (IFN) $\gamma$ in the culture supernatant was measured by ELISA (see below). This assay detects in vivo primed Th1, in that splenocytes from naive, nontransplanted mice produce minimal or undetectable concentrations of IFN $\gamma$ under these conditions $(13,14)$. To detect quiescent precursor Th1, splenocytes were stimulated for 5 days with irradiated donor strain splenocytes (15). Resulting cell populations were harvested, washed, and restimulated with irradiated donor cells for an additional $72 \mathrm{~h}$, and the concentration of IFN $\gamma$ in the culture supernatant was measured by ELISA.

\section{IFN ELISA}

Experimental samples $(100 \mu \mathrm{L})$ were added to ELISA plate wells that were coated with $5 \mu \mathrm{g} / \mathrm{mL}$ rat anti-mouse IFN $\gamma$ capture antibody (PharMingen, San Diego, CA, USA). Following a 1-h incubation at room temperature, plates were washed with Tween-20 (0.05\%) in PBS and incubated with $100 \mu \mathrm{L}$ of rat anti-mouse secondary biotinylated antibody $(1 \mu \mathrm{g} / \mathrm{mL})$ at room temperature for $45 \mathrm{~min}$. Plates were then washed, and $100 \mu \mathrm{L}$ of avidinperoxidase (Sigma Chemicals, St Louis, MO, USA) was added. Following a 30-min incubation at room temperature, plates were developed with ABTS (2,2'-azino-bis-3ethylbenzthiazoline-6-sulfonic acid) substrate (Sigma Chemicals, St Louis, MO, USA). After 20 min, absorbance was determined at $405 \mathrm{~nm}$ by an EL 800 microplate reader (Bio-Tek Instruments, Winooski, VT, USA). Sample cytokine concentrations were calculated from a standard curve that was derived from 2-fold serial dilutions of murine recombinant IFN $\gamma$. Data are reported as the mean and SD of IFN $\gamma$ values for 6-10 individual animals.

\section{Limiting dilution analysis (LDA) of donor-reactive IL-2 producing helper T lymphocytes (HTL)}

Two related LDA techniques were employed that distinguish in vivo primed IL-2 producing HTL, from quiescent precursor HTL. These assays have previously been described in detail (16). The LDA technique employed to detect in vivo primed cells is based on the principle that IL-2 production by primed HTL, but not quiescent precursor HTL, is resistant to irradiation (17). Hence, responder cells were irradiated (2000R) prior to addition to the LDA microcultures to quantify primed HTL. To detect precursor HTL, responder cells were not irradiated. Irradiated allogeneic splenocytes (5000 R) were then added as stimulator cells, the microcultures were overlaid with the IL-2-dependent cell line CTLL-20 $\left(1 \times 10^{3} /\right.$ well $)$, pulsed with ${ }^{3} \mathrm{H}$-thymidine, and harvested. ${ }^{3} \mathrm{H}$-thymidine incorporation was determined by scintillation (BetaPlate, Wallac, Gaithersburg, MD, USA). Individual microcultures were considered positive for $\mathrm{IL}-2$ production if ${ }^{3} \mathrm{H}$-thymidine 
incorporation exceeded the mean plus 3SD of ${ }^{3} \mathrm{H}$-thymidine incorporation in microcultures lacking responder cells.

\section{Limiting dilution analysis of donor-reactive precursor cytotoxic $T$ lymphocyte (CTL)}

LDA was employed to quantify donor-specific CTL, as described (18). Briefly, dilutions of responder cells were plated in the presence of irradiated (2000R) allogeneic stimulators cells in DMEM containing 20\% EL-4 supernatant as a source of growth factors and $10 \%$ FCS. Following a 7 day incubation, cytolytic activity was detected by a standard ${ }^{51} \mathrm{Cr}$ release assay utilizing Con A-stimulated $(1 \mu \mathrm{g} / \mathrm{mL})$ donor strain splenocytes as target cells. Microcultures were considered cytolytic if the observed chromium release exceeded the mean plus 3SD of the chromium release in wells that contained target cells, stimulator cells, but no responder cells.

\section{Limiting dilution analysis data analysis}

Minimal estimates of HTL or CTL frequency were obtained according to the Poisson distribution equation as the slope of a line relating the number of responder cells per microwell (plotted on a linear $x$ axis) and the percentage of microwells that failed to produce IL-2 or to develop cytolytic activity respectively (plotted on a logarithmic $y$ axis). The slope of this regression line was generated by computer using chi-square minimization analysis, as described (19). This analysis yields a minimal frequency estimate, 95\% confidence interval of the frequency estimate, and a chi-square estimate probability.

\section{Alloantibody determination}

P815 cells $\left(\mathrm{H}-2^{\mathrm{d}}\right)$ were stained for flow cytometric analysis using diluted $(1: 50)$ sera obtained from cardiac allograft recipients as the primary antibody, followed by FITC-conjugated isotype specific anti-mouse $\lg \mathrm{M}$, IgG1, or IgG2a secondary antibodies (The Binding Site, San Diego, CA, USA) used at a 1:50 dilution. Data are reported as the mean channel fluorescence determined on a Becton Dickinson FACSCaliber (San Jose, CA, USA)

The enzyme-linked immunospot (ELISPOT) assay for IL-4 producing cells

The ELISPOT assay used to quantify alloantigen primed IL-4 producing cells has been described (20). Capture and detection anti-IL-4 mAb were obtained from Pharmingen. Polyvinylidene fluoride bottomed plates (Jade Scientific, Canton, MI, USA) were coated overnight with capture anti-IL-4 $\mathrm{mAb}(11 . \mathrm{B} 11,2 \mu \mathrm{g} / \mathrm{mL})$, blocked for $90 \mathrm{~min}$ with $1 \%$ BSA in PBS at R.T. and washed 3 times with PBS. Irradiated (5000R) donor splenocytes $\left(4 \times 10^{5}\right)$ were added to each well followed by $1 \times 10^{6}$ recipient splenocytes. Plates were incubated for $24 \mathrm{~h}$ at $37^{\circ} \mathrm{C}$, washed 3 times with PBS, and washed 4 times with PBS-Tween-20 (0.05\%). Biotinylated detection antiIL-4 mAb (BZD6-24G2, $2 \mu \mathrm{g} / \mathrm{mL}$ ) was added to each well and incubated overnight at $4{ }^{\circ} \mathrm{C}$. Plates were washed 3 times with PBS-Tween-20, and

Table 1: Impact of inductive anti-CD40L therapy on allograft survival

\begin{tabular}{lll}
\hline Recipient & Survival in days & Mean \pm SD \\
\hline Unmodified & $7(n=4) .8(n=2)$ & $7.25 \pm 0.46^{*}$ \\
h-IgG treated & $7(n=2), 8(n=4)$ & $7.70 \pm 0.51^{*}$ \\
Anti-CD40L treated & $60+(n=60)$ & $>60^{* *}$ \\
\hline
\end{tabular}

C57BL/6 mice were transplanted with BALB/c cardiac allografts and were either left untreated, or given control hamster $\lg \mathrm{G}(\mathrm{h}-\lg \mathrm{G})$ or anti-CD40L mAb on days 0, 1 and 2 post transplantation. Transplant function was evaluated by daily palpation. $A$ ' + ' indicates that allografts were functioning at the time of harvest.

* Not significantly different.

** $\mathrm{p}<0.05$ compared to unmodified and $\mathrm{h}$-lgG treated recipients. horse-radish peroxidase conjugated Strepavidin $(1: 2000$ dilution, DAKO Corp., Carpinteria, CA, USA) was added for $90 \mathrm{~min}$ at R.T. Plates were washed 4 times with PBS, developed with $\mathrm{AEC}$, washed with $\mathrm{H}_{2} \mathrm{O}$, and air-dried. Spots were enumerated using an ImmunoSpot Series 1 ELISPOT Analyzer (Cellular Technology Ltd, Cleveland, OH, USA).

\section{Statistical analyses}

P-values were obtained for graft survival using the log-rank comparison, generated by Kaplan-Meier survival curves. P-values for other data were determined using one-way ANOVA analysis with Tukey-Kramer post-test. All statistical analysis was performed using GraphPad Software (San Diego, CA, USA).

\section{Results}

\section{Inductive anti-CD40L therapy markedly prolongs allograft survival}

Cardiac allograft survival was monitored in unmodified recipients, recipients given inductive, multiple dose anti-CD40L therapy, or control hamster $\lg G$ (h-lgG) antibodies (Table 1). The mean survival time for unmodified allograft recipients was 7.25 days, which was comparable to graft survival in recipients treated with h-lgG. In contrast, multiple-dose inductive anti-CD40L treatment resulted in uniform graft survival of greater than 60 days. Histological analysis of allograft rejection on day 7 in unmodified recipients revealed an intense mononuclear cell infiltrate, many dead and degenerating myocytes, inflamed arteries, and scattered areas of hemorrhage (Figure 1A). Allografts obtained from h-lgG-treated recipients on day 7 exhibited a similar histological picture (data not shown). In contrast, functioning allografts obtained from anti-CD40L-treated recipients on day 7 post transplantation contained a very mild, diffuse mononuclear cell infiltrate, viable myocytes and uninvolved arteries (Figure 1B).

\section{Inductive anti-CD40L treatment inhibits priming of IL-2 producing HTL}

In vivo priming of IL-2 producing HTL has previously been reported to be associated with acute allograft rejection in this model (21). Hence, we utilized LDA to monitor the impact of anti-CD40L therapy on priming of donor-specific IL-2 producing HTL (16). In vivo primed IL-2 producing HTL were readily detected in unmodified and h-IgG-treated allograft recipients on day 7 post transplantation, but were not detectable in recipients treated with inductive anti-CD40L therapy (Table 2). As anticipated, primed HTL were not detectable in naive, nontransplanted mice. Donor-specific precursor IL-2 producing HTL frequencies were also determined by LDA, as described (16). Precursor HTL frequencies were similar in all groups (Table2), indicating that anti-CD4OL therapy prevented HTL priming, but did not result in the deletion of donor-reactive HTL during this early post-transplant period.

\section{In vivo priming of IFN $\gamma$ producing Th 1}

In this model, in vivo sensitization of IFN $\gamma$-producing Th1 has also been reported to be associated with acute allograft rejection $(14,15)$. Hence, splenocytes from allograft recipients were cultured in vitro in the presence of irradiated donor- 

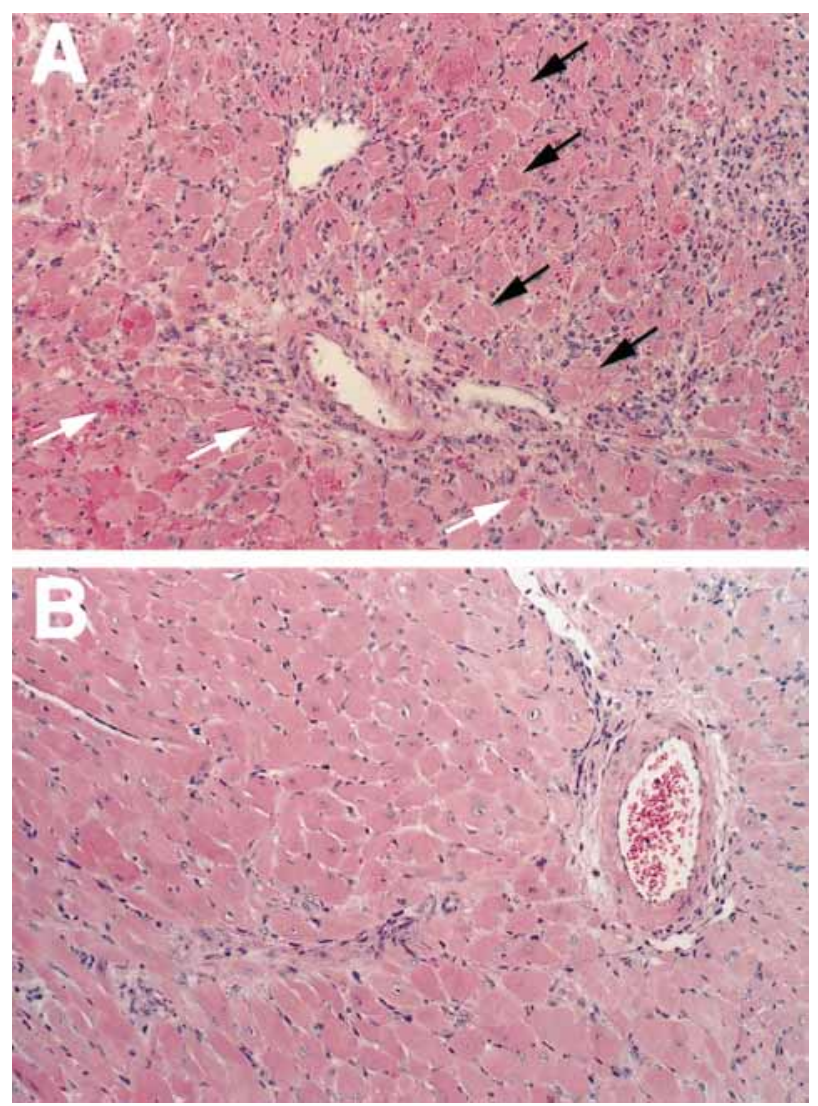

Figure 1: Anti-CD40L therapy prevents mononuclear cell infiltration and cardiac allograft damage. C57BL/6 mice were transplanted with BALB/c cardiac allografts and were either left untreated (Panel A) or given anti-CD4OL therapy (Panel B). On day 7 post transplantation, allografts were processed for H\&E staining. Unmodified rejection (Panel A, 200X) was characterized by an intense mononuclear cell infiltrate, inflamed arteries, scattered areas of hemorrhage (white arrows), and dead and degenerating myocytes (black arrows). In contrast, functioning allografts harvested from anti-CD40L treated recipients (Panel B, 200X) contained a very mild diffuse mononuclear cell infiltrate, myocytes remained viable, and arteries were unremarkable. Photomicrographs are representative of at least 6 individual transplants per group.
Table 3: Anti-CD40L therapy inhibits in vivo priming of IFN $\gamma$ producing Th1

\begin{tabular}{ll}
\hline Recipient reatment & Primed Th1 function \\
& IFN $(\mathrm{ng} / \mathrm{mL}) \pm: S D$ \\
\hline No transplant & $<0.39$ \\
Unmodified & $10.2 \pm 0.31$ \\
h-lgG treated & $6.6 \pm 0.47$ \\
Anti-CD40L treated & $<0.39^{*}$ \\
\hline
\end{tabular}

C57BL/6 mice were transplanted with BALB/c allografts and were either left untreated, or given control hamster lgG (h-lgG) or antiCD40L mAb on days 0,1 and 2 post transplantation. On day 7 post transplantation, recipient splenocytes were harvested and processed for use as responder cells in mixed lymphocyte cultures stimulated with irradiated BALB/c splenocytes. Naive C57BL/6 splenocytes obtained from nontransplanted mice served as negative controls for primed Th1 function. Supernatants were collected at $72 \mathrm{~h}$, and IFN $\gamma$ concentrations were determined by ELISA. Data are reported as the mean and SD of IFN $\gamma$ production of 6 individual transplant recipients in the $\mathrm{h}$-lgG-treated group and 10 individual animals in the other experimental groups. ${ }^{*} p<0.05$ compared to unmodified and h-lgG-treated recipients. Limit of detection for the ELISA $=0.39 \mathrm{ng} / \mathrm{mL}$

strain splenocytes for $72 \mathrm{~h}$, and culture supernatants were assessed for IFN $\gamma$ production (Table 3). This short-term stimulation of cells with donor alloantigens results in the release of IFN $\gamma$ from primed, but not quiescent precursor Th1 $(13,14)$. While splenocytes obtained from naive, nontransplanted mice did not produce measurable IFN $\gamma$ in this assay, significant IFN $\gamma$ production was observed by cells harvested from unmodified and control h-lgG-treated allograft recipients. In contrast, priming of IFN $\gamma$-producing Th1 was not observed in anti-CD40L-treated recipients.

\section{Anti-CD40L therapy inhibits donor-reactive alloanti- body production}

Donor-specific alloantibody responses have also been associated with acute allograft rejection in this model (22). Further, it is well established that CD40-CD40L interactions are crucial for antibody isotype switching, in that cells from animals deficient in either CD40 or CD40L fail to produce an

Table 2: Anti-CD40L therapy inhibits donor-specific IL-2 producing HTL priming during acute rejection

\begin{tabular}{lll}
\hline Recipient treatment & Primed HTL (95\% C.I.) & Precursor HTL (95\% C.I.) \\
\hline No transplant & $<1 / 189900$ & $1 / 8886(1 / 6188-1 / 15752)$ \\
Unmodified & $1 / 10157(1 / 6982-1 / 18628)$ & $1 / 7044(1 / 4935-1 / 12296)$ \\
h-IgG treated & $1 / 8162(1 / 5727-1 / 14199)$ & $1 / 11367(1 / 7736-1 / 21422)$ \\
Anti-CD4OL treated & $<1 / 189900$ & $1 / 84411 / 5929-1 / 14677)$ \\
\hline
\end{tabular}

C57BL/6 mice were transplanted with BALB/c allografts and were either left untreated, or given control hamster lgG (h-lgG) or anti-CD40L $\mathrm{mAb}$ on days 0,1 and 2 post transplantation. On day 7 post transplantation, recipient splenocytes were harvested and processed for use as responder cells in LDA to quantify primed and precursor IL-2 producing HTL (see Methods and Materials). Naive C57BL/6 obtained from nontransplanted mice served as negative controls for HTL priming. Data are presented as the frequency of primed and precursor IL-2 producing HTL and the 95\% confidence interval (95\% C.I.) of the frequency estimate. Data are representative of three separate experiments, consisting of 3 mice per experimental group. Frequencies with overlapping 95\% confidence intervals are not considered statistically different. Limit of detection for the LDA $=1 / 189900$. 
isotype switch $(23,24)$. Hence, donor-specific $\lg M, \lg G 1$ and $\lg \mathrm{G} 2 \mathrm{a}$ antibodies were monitored in the sera of allograft recipients (Table 4). While sera alloantibodies were readily detectable in unmodified and h-lgG-treated allograft recipients, they were not detected in anti-CD40L-treated recipients. It should also be noted that CD4+ T-cell independent IgM response (22) was also prevented by anti-CD40L therapy (Table 4).

\section{Inductive anti-CD40L therapy delays signs of chronic rejection}

While other immunosuppressive agents may prolong allograft survival, chronic rejection generally develops in long-term cardiac allografts $(15,25,26)$, which may be observed by day 60 post transplant. Cardiac allografts that were harvested on day 60 following inductive anti-CD40L therapy did not exhibit overt signs of chronic rejection such as graft fibrosis and neointimal development. Myocytes remained viable (Figure 2A), and collagen deposition was minimal as assessed by trichrome staining (Figure 2B). Further, neointimal development was absent as assessed by elastin staining (Figure 2C), and arteries remained patent. Hence, multiple-dose anti-CD4OL therapy appeared to delay the onset of chronic rejection relative to other immunosuppressive therapies that prolong graft survival.

\section{Precursor donor-reactive cells are maintained in re- cipients bearing long-term cardiac allografts}

Limiting dilution analysis was performed on splenocytes from mice bearing long-term cardiac allografts to assess whether graft acceptance was associated with a deletion of donor alloantigen-reactive T cells (Table 5). Precursor donor-reactive IL-2 producing HTL and CTL frequencies were similar in mice bearing long-term cardiac allografts to those observed in naive, nontransplanted animals. To determine if precursor

Table 4: Anti-CD40L therapy inhibits the production of donor-reactive alloantibodies

\begin{tabular}{llll}
\hline Recipient treatment & \multicolumn{3}{l}{ Mean channel fluorescence } \\
\cline { 2 - 4 } & $\operatorname{lgM}$ & $\operatorname{lgG} 1$ & $\operatorname{lgG} 2 \mathrm{a}$ \\
\hline No transplant & 3.6 & 2.7 & 3.0 \\
Unmodified & 58.6 & 8.3 & 10.0 \\
h-IgG treated & 21.3 & 5.0 & 15.0 \\
Anti-CD4OL treated & 4.4 & 2.4 & 2.6 \\
\hline
\end{tabular}

$\mathrm{C} 57 \mathrm{BL} / 6$ mice were transplanted with BALB/c allografts and were either left untreated, or given control hamster $\operatorname{lgG}$ (h-lgG) or antiCD4OL mAb on days 0, 1 and 2 post transplantation. Sera obtained from naive, nontransplanted mice served as negative controls. $\mathrm{H}-2^{\mathrm{d}}$ specific alloantibodies were assessed by incubating diluted $(1: 50)$ control sera or sera obtained from animals 7 days post transplantation with P815 $\left(\mathrm{H}-2^{\mathrm{d}}\right)$ target cells, followed by FITC-conjugated isotype specific secondary antibodies (see Materials and Methods). Donor-reactive alloantibodies were determined by FACS analysis and reported as mean channel fluorescence. The mean channel fluorescence of isotype control secondary antibodies was 2.8 ( $\operatorname{lgM}$ ), 3.1 (lgG1) and 2.9 (lgG2a). Data are representative of 3 separate experiments consisting of 3 mice per experimental group.
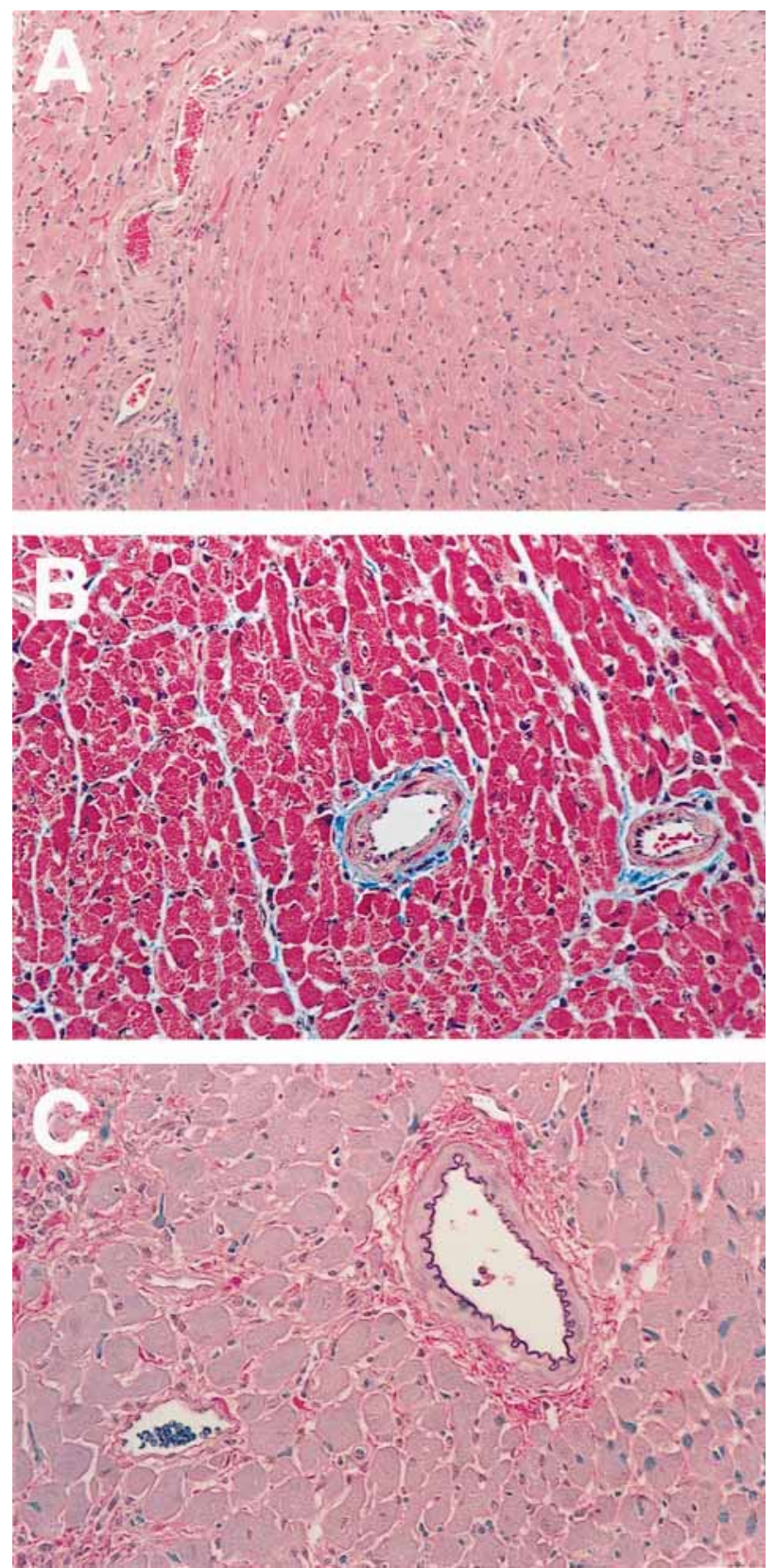

Figure 2: Inductive anti-CD40L therapy delays the development of chronic allograft rejection. $\mathrm{C} 57 \mathrm{BL} / 6$ mice were transplanted with BALB/c cardiac allografts and given anti-CD40L therapy. On day 60 post transplantation, functioning allografts were processed for histologic analysis. H\&E staining (Panel A, 200×) revealed viable myocytes and normal myocardial architecture. Trichrome staining (Panel B, 200X) revealed minimal collagen deposition (blue staining) within the myocardium and the normal collagen component of the arterial adventitia. Elastin staining (Panel C, $400 \times$ ) revealed near total absence of neointima development. Photomicrographs are representative of at least 10 individual transplants. 


\section{Nathan et al.}

Table 5: Inductive anti-CD40L therapy does not result in the deletion of donor alloantigen-reactive T cells in mice bearing long-term cardiac allografts

\begin{tabular}{|c|c|c|c|c|}
\hline Recipient treatment & $\begin{array}{l}\text { Precursor HTL } \\
\text { (95\% C.I.) }\end{array}$ & $\begin{array}{l}\text { Precursor CTL } \\
\text { (95\% C.I.) }\end{array}$ & $\begin{array}{l}\text { Precursor Th1 } \\
\text { IFN } \gamma(\mathrm{ng} / \mathrm{mL})\end{array}$ & $\begin{array}{l}\text { IL-4 producing Th2 } \\
\# / 10^{6} \text { cells) }\end{array}$ \\
\hline No transplant & $\begin{array}{l}1 / 5709 \\
(1 / 4083-1 / 9950)\end{array}$ & $\begin{array}{l}1 / 1841 \\
(1 / 1326-1 / 3011)\end{array}$ & ${ }^{*} 6.7 \pm 1.28$ & ** $10.3 \pm 8.5$ \\
\hline Long-term anti-CD40L & $\begin{array}{l}1 / 9045 \\
(1 / 6185-1 / 16824)\end{array}$ & $\begin{array}{l}1 / 1453 \\
(1 / 1077-1 / 2222)\end{array}$ & $* 4.5 \pm 0.25$ & $* * 18.0 \pm 5.6$ \\
\hline
\end{tabular}

C57BL/6 mice were transplanted with BALB/c allografts and given inductive anti-CD40L therapy. On day 60 post transplantation, splenocytes were harvested for use in donor-specific T-cell functional assays. Responses are compared to those mounted by splenocytes obtained from naive, nontransplanted mice. The frequencies of precursor IL-2 producing helper cells and cytotoxic T cells were determined by LDA, and the data are reported as the minimal frequency estimate and 95\% confidence interval. Precursor Th1 function was assessed as described in Materials and Methods, and the data are presented as the mean and SD of IFN $\gamma$ release. IL-4 producing cells were quantified by ELISPOT, and the data are reported as the number of spots per $10^{6}$ cells. Results are representative of at least 6 individual animals.

$*$ * ${ }^{* *}$ Not significantly different.

Th1 were deleted in mice bearing long-term cardiac allografts, splenocytes were stimulated for 5 days with irradiated donor splenocytes, washed, and restimulated with donor antigens for an additional $72 \mathrm{~h}$, as described (15). In this precursor Th1 assay, IFN $\gamma$ release was comparable when splenocytes were obtained from mice bearing long-term cardiac allografts or from naive mice. Hence, donor-reactive precursor HTL, CTL and Th1 responses in mice bearing long-term allografts were similar to those observed in naive mice, indicating that these cells had not been deleted. Since costimulatory blockade has previously been reported to induce do-

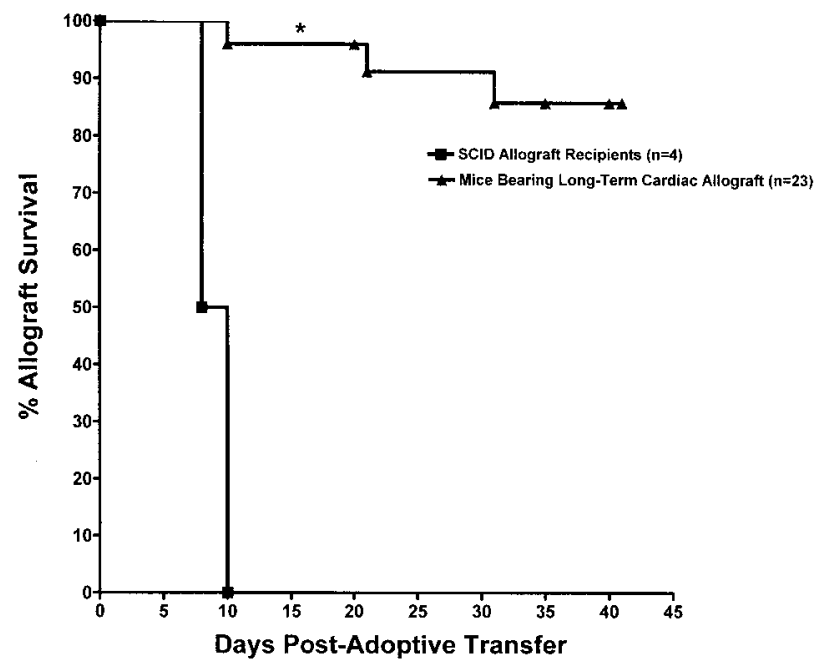

Figure 3: Differentiated effector cells are incapable of mediating rejection of long-term cardiac allografts. Unmodified C57BL/6 mice were transplanted with BALB/c allografts. On day 10 post transplantation, recipient spleens were harvested, pooled and processed into single-cell suspensions. $5 \times 10^{7}$ splenocytes were adoptively transferred i.v. into SCID allograft recipients 5 days post transplantation, or into mice treated with inductive anti-CD40L therapy that were bearing functioning allografts on day 60. Transplant function was monitored by daily palpation. ${ }^{*} \mathrm{p}<0.05$. nor-reactive Th2 (27), we employed an ELISPOT assay to quantify IL-4 producing Th2 responses (Table 5). We have reproducibly used this assay to quantify IL-4 producing cells under conditions that favor in vivo Th2 development (28). However, IL-4 producing Th2 responses were similar in mice bearing long-term cardiac allografts and naive animals, indicating that inductive anti-CD40L treatment did not preferentially induce donor alloantigen-reactive Th2 responses.

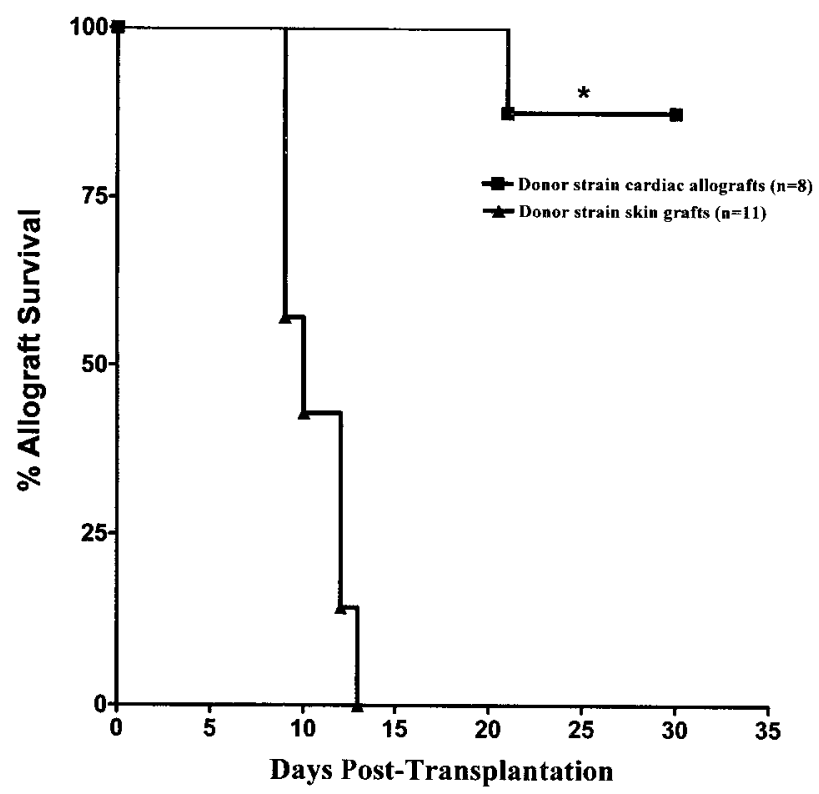

Figure 4: Induction of cardiac, but not skin allograft acceptance by anti-CD40L therapy. C57BL/ 6 mice were transplanted with BALB/c cardiac allografts and given anti-CD40L therapy. On day 60 post transplantation, mice were grafted with BALB/c skin or were transplanted with a second BALB/c cardiac allograft placed in the cervical position. Skin allograft $(n=11)$ and secondary cardiac allograft $(n=8)$ survival times are reported. All primary cardiac allografts continued to function normally for the duration of the experiment. ${ }^{*} p<0.05$ for skin allograft vs. secondary cardiac allograft survival.

American Journal of Transplantation 2002; 2: 323-332 


\section{Differentiated effector cells are incapable of mediating graft rejection in mice bearing long-term cardiac allo- grafts}

Since donor alloantigen-reactive cells were maintained in a quiescent state in mice bearing long-term allografts, we asked whether fully differentiated effector cells could mediate rejection of these grafts. To this end, splenocytes were obtained from unmodified allograft recipients on day 10, after they had rejected their allografts. These effector cells were adoptively transferred into mice bearing long-term (day 60) cardiac allografts, or into SCID allograft recipients (Figure 3). While SCID recipients of these effector cells readily rejected their allografts (mean graft survival $=10.1$ days), the vast majority of mice bearing long-term cardiac allografts retained functioning grafts as long as 40 days following the adoptive transfer of effector cells. These observations are consistent with the presence of regulatory cells that are capable of silencing effector cell function.

\section{Mice bearing long-term cardiac allografts reject donor strain skin grafts, but not secondary cardiac grafts}

To further characterize the state of allograft acceptance following inductive anti-CD40L therapy, mice bearing long-term cardiac allografts were grafted with either donor-strain skin allografts (Figure 4) or isografts (data not shown). Skin allografts, but not isografts, were uniformly rejected by day 13 post-skin grafting. However, the original cardiac allografts continued to function in mice that had rejected the donorstrain skin grafts. Histological analyses of these cardiac allografts revealed only minimal or no changes relative to the long-term allografts depicted in Figure 2 (data not shown). At least two possibilities could explain these observations: (i) The original cardiac allograft had lost its immunogenicity with time in the recipient, or (ii) a state of tissue-specific allograft hyporesponsiveness had been established. To discriminate between these possibilities, mice bearing long-term cardiac allografts were transplanted with a second donor-strain cardiac allograft in the cervical position (Figure 4). Interestingly, $7 / 8$ of these mice failed to reject their second donor-strain cardiac allograft, and all mice retained their original abdominal allograft. Histologic examination revealed that both the primary abdominal and the secondary cervical cardiac allografts remained free of overt tissue damage (Figure 5). Collectively, these results argue against the loss of allograft immunogenicity as an explanation for long-term graft acceptance. Further, they suggest that a state of tissue-specific allograft acceptance had been induced.

\section{Discussion}

Although several groups have reported that blockade of CD40-CD40L interactions results in prolonged allograft survival (29-33), the mechanism by which this occurs has not yet been fully defined. Hence, the focus of this study was to provide further insight into the immunologic parameters that are influenced by anti-CD40L therapy and that are associated with long-term allograft acceptance. While several studies

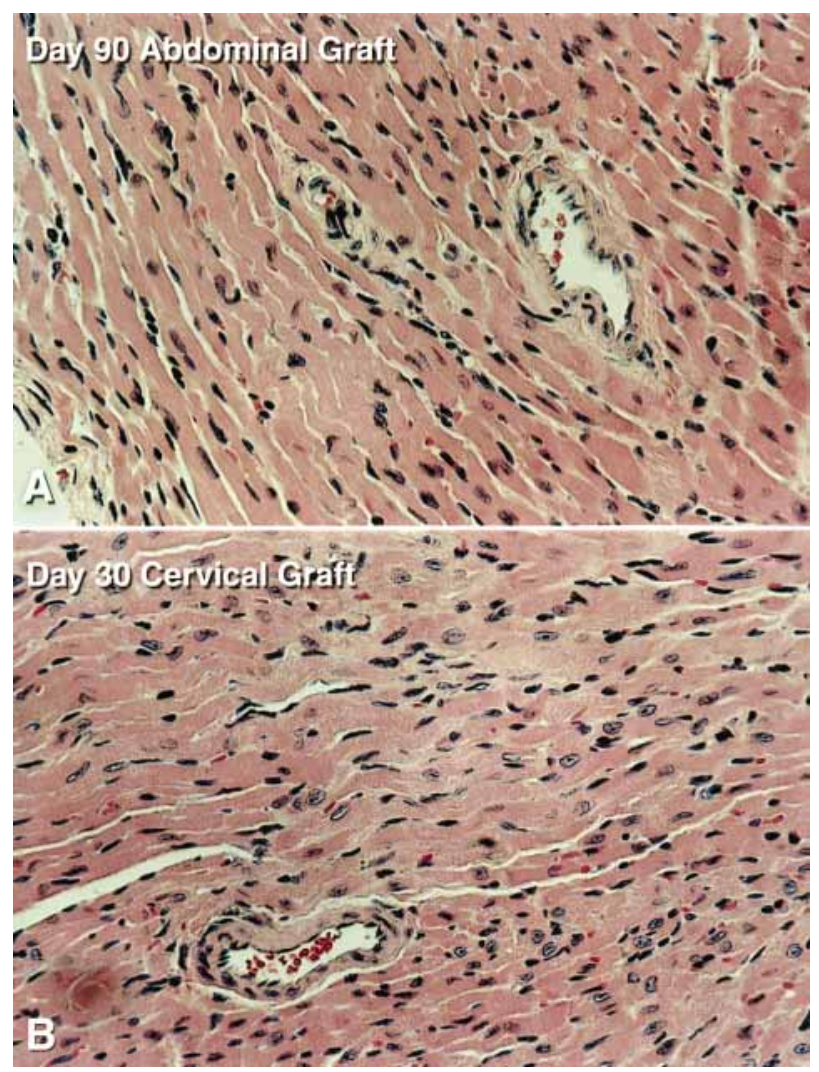

Figure 5: Histology of primary and secondary cardiac allografts following inductive anti-CD40L therapy. C57BL/6 mice were transplanted with BALB/c cardiac allografts and given antiCD40L therapy. On day 60 post transplantation, mice were transplanted with a second BALB/c cardiac allograft placed in the cervical position. Thirty days later, both the primary abdominal (Panel A) and the secondary cervical (Panel B) cardiac allografts were harvested and processed for H\&E staining. In both cases, allografts exhibited normal myocardial architecture with viable myocytes, uninvolved arteries, and a mild diffuse mononuclear cell infiltrate $(400 \times)$. Photomicrographs are representative of allografts obtained from 7 individual transplant recipients.

have shown that anti-CD40L therapy is less effective when used alone $(29,30,33,34)$, we found that three 1 -mg doses of anti-CD40L mAb as a sole immunosuppressant resulted in uniform long-term acceptance of cardiac allografts (Table 1). The enhanced protective activity of this higher-dose anti-CD40L therapy used in our study is likely due to distinct dosing regimens employed by these previous studies. The increased dose of anti-CD40L did not result in noticeable side-effects and appeared to dissipate from the circulation, in that mice transplanted with allografts 28 days following the initiation of therapy acutely rejected their grafts (data not shown). Nonetheless, our data demonstrate that long-term allograft acceptance following inductive anti-CD40L therapy may be achieved in the continued presence of donor alloantigen-reactive $T$ cells (Table5). Hence, inductive antiCD40L therapy did not result in the deletion of donor-reactive $\mathrm{T}$ cells, but allowed these cells to be maintained in numbers similar to those found in naive mice. 


\section{Nathan et al.}

The mechanism involved in maintaining donor-reactive T cells in a quiescent state remains to be established. Waldmann and colleagues (10) have recently demonstrated that inductive anti-CD40L therapy promotes the development of cells that are capable of mediating infectious tolerance when naive precursor cells are adoptively transferred into to mice bearing long-term skin allografts. Further, the current study revealed that infusion of differentiated effector cells obtained from mice that had rejected cardiac allografts were incapable of rejecting long-term cardiac allografts following inductive antiCD40L therapy (Figure 3). Collectively, these data suggest that anti-CD40L therapy induces regulatory cells that are capable of preventing precursor as well as differentiated effector cells from mediating graft rejection. The finding that mice bearing long-term cardiac allografts were capable of rejecting donor-strain skin grafts, but not secondary cardiac allografts (Figures 4 and 5) suggests that these putative regulatory cells may function in a tissue-specific manner. Indeed, the nature of a primary allograft has been shown to dramatically influence the fate of subsequent transplants (35). It should be noted that cardiac allograft rejection is accompanied by the development of cardiac myosin-specific effector cells (36). Furthermore, the presence of cardiac myosin-reactive antibodies has been associated with an increase in the frequency and severity of cardiac allograft rejection episodes in man (37). Similarly, Sachs and coworkers have recently identified skin-specific antigens that are not expressed in cardiac or hematopoietic tissue (38). Hence, skin-specific effector cells may be induced by skin grafting that would not be induced by a second cardiac graft. It is also conceivable that the putative regulatory cells involved in anti-CD40L-induced cardiac allograft acceptance may, in part, be cardiac myosin specific. In addition to this notion of tissue-specific regulatory cells, other possibilities should be considered. For example, tissue-specific effector cells may be either deleted or altered in function. These alterations in tissue-specific effector cells would not be detected by the assays employed in our study. Further, the nature and content of APC in the skin and heart may impact on tissue immunogenicity and graft survival. Differences in skin and second cardiac allografts survival may also be influenced by the nature of the lymphoid organs that drain these grafts.

Anti-CD40L therapy inhibited a number of immune parameters that have been historically associated with acute allograft rejection, including the production of both $\operatorname{lgM}$ and IgG alloantibodies (Table 4), and the priming of Th1 (Table 3) and IL-2 producing HTL (Table 2). It should be noted that lower-dose anti-CD40L therapy does not prevent intragraft cytokine gene expression (29), suggesting that T-cell priming may not be prevented by low-dose regimens. Several of these observations suggest that immune responses that are modified by anti-CD40L therapy may be distinct from responses that occur in mice that are deficient in CD4OL (CD40L -/-). For example, CD40L -/- mice are capable of mounting IgM antibody responses (24). However, treatment of cardiac allograft recipients with anti-CD40L mAb abrogated the production of IgM alloantibodies (Table 4). It should be noted that prolonged renal allograft survival in nonhuman primates treated with a humanized anti-CD40L mAb was associated with the delayed (30-60 days) development of low titer donor-reactive lgG, but not lgM antibodies (32). The delayed production of these lgG antibodies had no impact on transplant survival, and these antibodies were not deposited in the allograft at detectable levels. In addition, CD40L -/mice have been shown to mount normal Th1 responses when challenged with Histoplasma capsulatum (39). However, we found that anti-CD40L therapy prevented Th1 priming in response to cardiac transplantation (Table 3). This observation may be related to anti-CD40L's inhibitory effects on IL-12 production (40). Finally, while CD40L -/- mice accept cardiac allografts, the development of chronic graft arteriosclerosis is not prevented in these mice and is readily detectable by 8 weeks post transplant (41). Allografts in CD40L -/recipients are also infiltrated by mononuclear cells and suffer significant parenchymal cell damage (41). In our hands, multiple dose anti-CD40L therapy abrogated mononuclear cell infiltration, arteriosclerosis, and fibrosis of the graft (Figure 2), as assessed on day 60 post transplant. Others have reported similar observations when anti-CD40L mAb is used in conjunction with CTLA4-lg (29). It should also be noted that CD4OL appears to be necessary for the progression of nontransplant-associated atherosclerosis (42). Hence, clear differences have been reported when CD40-CD40L interactions are prevented by CD40L deficiency vs. treatment with anti-CD4OL mAb. Some of the above differences in CD40L -/- mice and animals treated with anti-CD40L mAb may be due in part to variables in distinct experimental systems. However, these discrepancies may also reflect the direct immunomodulatory effects that anti-CD40L mAb has on T cells (7).

It has been proposed that long-term allograft acceptance may be facilitated by immune deviation towards Th2 dominance (43). While long-term graft acceptance resulting from blockade of the CD28-B7 pathway has been associated with persistent Th2 responses (27), we were unable to detect appreciable numbers of donor-reactive $\mathrm{IL}-4$ producing cells in mice given inductive anti-CD40L therapy (Table 5). In keeping with our observations, Larsen et al. (29) found that simultaneous blockade of the CD28-B7 and CD40-CD40L pathways inhibited both Th1 and Th2 gene expression. It should be noted that a growing body of evidence indicates that the Th2 cytokines IL-4 and IL-5 may contribute to allograft rejection (43). Hence, sparing or preferential induction of Th2 by inductive anti-CD40L therapy could have a detrimental impact on long-term allograft survival. However, these observations do not rule out a potential role for other Th2- or Th3associated cytokines, such as IL-10 and TGF $\beta$, in anti-CD40L therapy-induced allograft acceptance. It is well established that IL-10 (44) or TGF $\beta$ (45) producing regulatory cells are involved in the control of immune-mediated pathology in a variety of disease states. In addition to the well-accepted role of regulatory cells in regulating Th1 responses $(44,45)$, IL-10 producing regulatory cells have recently be shown to inhibit Th2 responses in vivo (46). Further, Orosz's group has dem- 
onstrated that both IL-10 and TGF $\beta$ contribute to the regulation of DTH responses in mice bearing long-term cardiac allografts (47). The role of these anti-inflammatory cytokines in anti-CD40L-induced graft acceptance remains to be established.

In summary, our data indicate that deletion of donor alloantigen-reactive $T$ cells, as assessed by a variety of in vitro assays, is not necessary for long-term allograft acceptance following inductive anti-CD40L therapy. Rather, our data are in keeping with the recent report by Waldmann and coworkers (10) that implicates regulatory cell development as a critical feature in anti-CD40L-induced graft acceptance. It is also possible that the cardiac allograft-specific tolerance reported herein may be explained, in part, by a failure of antiCD40L-treated mice to develop cardiac myosin-specific effector cells (36) or by the induction of cardiac myosin-specific regulatory cells. These possibilities are currently being investigated.

\section{Acknowledgments}

We thank Drs Kewang Li, Guanyi Lu, and Ying Wang for their excellent microsurgical assistance. We also thank Dr Anita Chong for her helpful discussions and Dr Randy Noelle for the gift of the MR1 secreting hybridoma.

This work was supported by R01 Al/HL 31946 (DKB) from the National Institutes of Health

\section{References}

1. Schwartz RH. A cell culture model for T lymphocyte clonal anergy. Science 1990; 248: 1349-1356

2. Ferguson TA, Green D. T cells are just dying to accept grafts. Nat Med 1999; 5: 1231-1232.

3. Li XC, Wells AD, Strom TB, Turka LA. The role of $T$ cell apoptosis in transplantation tolerance. Curr Opin Immunol 2000; 12: 522-527.

4. Grewal IS, Flavell RA. A central role of CD40 ligand in the regulation of CD4+ T-cell responses. Immunol Today 1996; 17: 410.

5. Gudmundsdottir $H$, Turka LA. T cell costimulatory blockade: New therapies for transplant rejection. J Am Soc Nephrol 1999; 10: 13561365.

6. Harlan DM, Kirk AD. The future of organ and tissue transplantation: Can T-cell costimulatory pathway modifiers revolutionize the prevention of graft rejection? JAMA 1999; 282: 1076-1082.

7. Blair PJ, Riley JL, Harlan DM et al. CD40 ligand (CD154) triggers a short-term CD4+ T cell activation response that results in a secretion of immunomodulatory cytokines and apoptosis. J Exp Med 2000; 191: 651-660

8. Iwakoshi NN, Mordes JP, Markees TG, Phillips NE, Rosini AA, Greiner DL. Treatment of allograft recipients with donor-specific transfusion and anti-CD154 antibody leads to deletion of alloreactive CD8+ T cells and prolonged graft survival in a CTLA4-dependent manner. $J$ Immunol 2000; 164: 512-521.

9. Wells $A D$, Li XC, Li $Y$ et al. Requirement for T-cell apoptosis in the induction of peripheral transplantation tolerance. Nat Med 1999; 5 1303-1307.

10. Graca L, Honey K, Adams E, Cobbold SP, Waldmann H. Anti-CD154 therapeutic antibodies induce infectious transplantation tolerance. $J$ Immunol 2000; 165: 4783-4786.
11. Corry R, Winn HJ, Russell PS. Primarily vascularized allografts of hearts in mice. The role of $\mathrm{H}-2 \mathrm{D}, \mathrm{H}-2 \mathrm{~K}$, and non- $\mathrm{H}-2$ antigens in rejection. Transplantation 1973; 16: 343-350.

12. Chen Z. A technique of cervical heterotopic heart transplantation in mice. Transplantation 1991; 52: 1099-1101.

13. Piccotti JR, Chan SY, Li K, Eichwald EJ, Bishop DK. Differential effects of IL-12 receptor blockade with IL-12 p40 homodimer on the induction of $\mathrm{CD} 4+$ and $\mathrm{CD} 8+\mathrm{IFN}-\gamma$-producing cells. J Immunol 1997; 158: 643-648.

14. Piccotti JR, Li K, Chan SY, Eichwald EJ, Bishop DK. Alloantigen-reactive Th1 helper T lymphocyte development in IL-12 deficient mice. J Immunol 1998; 160: 1132-1138.

15. Piccotti JR, Li K, Chan SY, Eichwald EJ, Bishop DK. Cytokine regulation of chronic cardiac allograft rejection: evidence against a role for Th1 in the disease process. Transplantation 1999; 67: 1548-1555.

16. Bishop DK, Orosz CG. Limiting dilution analysis for alloreactive, TCGFsecreting T cells: LDA methods that discriminate between unstimulated precursor T cells and in vivo alloactivated T cells. Transplantation 1989; 47: 671-677.

17. Glasebrook AL, Fitch FW. Alloreactive cloned T cell lines: interactions between cloned amplifier and cytolytic T cell lines. J Exp Med 1980; 151: 876-895

18. Orosz CG, Horstemeyer B, Zinn NE, Bishop DK. Development and evaluation of a limiting dilution analysis technique that can discriminate in vivo alloactivated cytotoxic $T$ lymphocytes from their native precursors. Transplantation 1989; 47: 189-194.

19. Taswell C. Limiting dilution assays for the determination of immunocompetent cell frequencies. J Immunol 1981; 126: 1614-1619.

20. Matesic D, Lehmann PV, Heeger PS. High resolution characterization of cytokine-producing alloreactivity in naive and allograft-primed mice. Transplantation 1998; 65: 906-914.

21. Bishop DK, Shelby J, Eichwald EJ. Mobilization of T lymphocytes following cardiac transplantation: evidence that CD4-positive cells are required for cytotoxic $\mathrm{T}$ lymphocyte activation, inflammatory endothelial development, graft infiltration, and acute allograft rejection. Transplantation 1992; 53: 849-857.

22. Bishop DK, Li W, Chan SY, Ensley RD, Shelby J, Eichwald EJ. Helper T lymphocyte unresponsiveness to cardiac allografts following transient depletion of CD4-positive cells: implications for cellular and humoral responses. Transplantation 1994; 58: 576-584.

23. Kawabe T, Naka T, Yoshida $\mathrm{K}$ et al. The immune responses in CD40deficient mice: impaired immunoglobulin class switching and germinal center formation. Immunity 1994; 1: 167-178.

24. Renshaw BR, Fanslow WC III, Armitage RJ et al. Humoral immune responses in CD40 ligand-deficient mice. J Exp Med 1994; 180: 1889-1900.

25. Schmid C, Heemann U, Tilney NL. Factors contributing to the development of chronic rejection in heterotopic rat heart transplantation. Transplantation 1997; 64: 222-228.

26. Orosz CG, Wakely E, Bergese SD et al. Prevention of murine cardiac allograft rejection with gallium nitrate: comparison with anti-CD4 monoclonal antibody. Transplantation 1996; 61: 783-791.

27. Sayegh MH, Akalin E, Hancock WW et al. CD28-B7 blockade after alloantigenic challenge in vivo inhibits Th1 cytokines but spares Th2. J Exp Med 1995; 181: 1869-1874.

28. Bishop DK, Wood SC, Eichwald EJ, Orosz CG. Immunobiology of allograft rejection in the absence of IFN $\gamma$ : CD8 + effector cells develop independently of CD4+ cells and CD40-CD40 ligand interactions. J Immunol 2001: 166: 3248-3255.

29. Larsen CP, Elwood ET, Alexander DZ et al. Long-term acceptance of skin and allografts after blocking CD40 and CD28 pathways. Nature 1996; 381: 434-438

30. Markees TG, Phillips NE, Noelle RJ et al. Prolonged survival of mouse skin allografts in recipients treated with donor splenocytes and antibody to CD40 ligand. Transplantation 1997; 64: 329-335. 


\section{Nathan et al.}

31. Bumgardner GL, Li J, Heininger M, Orosz CG. Costimulation pathways in host immune responses to allogeneic hepatocytes. Transplantation 1998; 66: $1841-1845$

32. Kirk AD, Burkly LC, Batty DS et al. Treatment with humanized monoclonal antibody against CD154 prevents acute renal allograft rejection in non-human primates. Nat Med 1999; 5: 686-693.

33. Sun $\mathrm{H}$, Subbotin $\mathrm{V}$, Chen $\mathrm{C}$ et al. Prevention of chronic rejection in mouse aortic allografts by combined treatment with CTLA4-Ig and anti-CD40 ligand monoclonal antibody. Transplantation 1997; 64: 1838-1856.

34. Lu L, Li W, Fu F et al. Blockade of the CD40-CD40 ligand pathway potentiates the capacity of donor-derived dendritic cell progenitors to induce long-term cardiac allograft survival. Transplantation 1997; 64: 1808-1815.

35. Vriens PW, Nisco SJ, Hoyt EG et al. Tissue-specific differences in the establishment of tolerance: tolerogenic effects of lung allografts in rats. Transplantation 1994; 57: 1795-1798.

36. Fedoseyeva EV, Zhang F, Orr PL, Levin D, Buncke HJ, Benichou G. De novo autoimmunity to cardiac myosin after heart transplantation and its contribution to the rejection process. J Immunol 1999; 162: 68366842.

37. Warraich RS, Pomerance A, Stanley A, Banner NR, Dunn MJ, Yacoub $\mathrm{MH}$. Cardiac myosin autoantibodies and acute rejection after heart transplantation in patients with dilated cardiomyopathy. Transplantation 2000; 69: 1609-1617.

38. Fuchimoto $Y$, Gleit AL, Huang $C A$ et al. Skin-specific alloantigens in miniature swine. Transplantation 2001; 72: 122-126.
39. Zhou P, Seder RA. CD40 ligand is not essential for induction of type 1 cytokine responses or protective immunity after primary or secondary infection with histoplasma capsulatum. J Exp Med 1998; 8: 13151324.

40. Stuber E, Strober W, Neurath M. Blocking the CD40L-CD40 interactions in vivo specifically prevents the priming of $\mathrm{T}$ helper 1 cells through the inhibition of interleukin 12 secretion. J Exp Med 1996; 2: 693-698.

41. Shimizu K, Schönbeck U, Mach F, Libby P, Mitchell PN. Host CD40 ligand deficiency induces long-term allograft survival and donor-specific tolerance in mouse cardiac transplantation but does not prevent graft arteriosclerosis. J Immunol 2000; 165: 3506-3518.

42. Lutgens EL, Gorelik MJ, Daemen AP et al. Requirement for CD154 in the progression of atherosclerosis. Nat Med 1999; 5: 1313-1316.

43. Piccotti JR, Chan SY, VanBuskirk AM, Eichwald EJ, Bishop DK. Are Th2 helper T lymphocytes beneficial, deleterious, or irrelevant in promoting allograft survival? Transplantation 1997; 63: 619-624.

44. Fowler S, Powrie F. Control of immune pathology by IL-10-secreting regulatory T cells. Springer Semin Immunopathol 1999; 21: 287-294.

45. Letterio JJ, Roberts AB. Regulation of immune responses by TGF- $\beta$. Annu Rev Immunol 1998; 16: 137-161.

46. Cottrez F, Hurst SD, Coffman RL, Grouz H. T regulatory cells 1 inhibit a Th2-specific response in vivo. J Immunol 2000; 165: 4848-4853.

47. Bickertsaff AA, VanBuskirk AM, Wakely E, Orosz CG. Transforming growth factor-beta and interleukin 10 subvert alloreactive delayed hypersensitivity in cardiac allograft acceptor mice. Transplantation 2000; 69: $1517-1520$ 